\title{
AEM Investigation of Strontium Substituted La-Co-Perovskites
}

\author{
I. Letofsky-Papst*, W. Grogger*, I. Rom*, F. Hofer*, E. Bucher**, W. Sitte** \\ * Research Institute for Electron Microscopy, Graz University of Technology, Steyrergasse 17, A- \\ 8010 Graz, Austria \\ ** Institute of Physical Chemistry, University of Leoben, Franz Josef Strasse 18, A-8700 Leoben, \\ Austria
}

Due to their high ionic conductivity strontium-substituted lanthanum cobaltites $\left(\mathrm{La}_{1-\mathrm{x}} \mathrm{Sr}_{\mathrm{x}} \mathrm{CoO}_{3-\delta}\right)$ have potential applications as solid oxide fuel cell cathodes, gas separation membranes or oxygen sensors. In the heavily Sr doped materials, the ionic conductivity obtained from galvanostatic polarization experiments shows a distinct maximum at certain $\delta$ values [1]. This behavior at high oxygen non-stoichiometry may be explained by oxygen vacancy ordering thus decreasing the mobility of the vacancies [2].

In order to confirm the assumption of vacancy ordering, two powders with $\mathrm{x}=0.6$ and different oxygen concentrations are investigated by TEM: LSC $(3-\delta \approx 3)$ and LSC $+(3-\delta=2.71)$. LSC was annealed at $1400^{\circ} \mathrm{C}$ and slowly cooled to room temperature to yield near-stoichiometric composition. The higher oxygen vacancy concentration in LSC + was reached by equilibrating the annealed material at $\mathrm{p}\left(\mathrm{O}_{2}\right)=10^{-3}$ bar for $60 \mathrm{~h}$ at $825^{\circ} \mathrm{C}$ and subsequent quenching to room temperature.

Looking at the elemental distributions, EFTEM reveals that both samples are perfectly homogenous at a nanometer scale [3]. The bright field image of LSC+ in Fig. 1a shows a single particle with a typical domain structure, i.e. bright and dark regions, which is not found in LSC. The dimension of these domains is between 50 and $200 \mathrm{~nm}$. The electron diffraction pattern along the [4 $2-1$ ] zone axis (hexagonal description) shows additional reflections which originate from these two sets of domains (Fig. 1b, see arrows). The extra spots may be explained by a superstructure. In order to resolve a possible difference between the two domains, HRTEM images of the domain boundary of LSC+ particles in the same orientation are acquired (Fig. 2). The insets in the HRTEM image are Fourier transforms (FFTs) of two adjacent domains, which are rotated about $90^{\circ}$ (in the image plane) with respect to each other and exhibit a doubling of the lattice spacing. These spatially resolved superstructure spots (arrows in Fig. 2) correspond to the extra spots in the SAED pattern (Fig. 1b). The near-edge fine structure of the oxygen $\mathrm{K}$ ionization edge looks significantly different for both samples (Fig. 3), which agrees well with the assumption that the superstructure is due to the ordering of oxygen vacancies thus changing the chemical surrounding of the oxygen atoms.

By these AEM investigations the occurrence of a superstructure in LSC + is confirmed, which is presumably due to the ordering of oxygen vacancies. This ordering is responsible for the decrease of the ionic conductivity with increasing oxygen vacancy concentration.

[1] E. Bucher et al., Solid State Ionics (in print)

[2] R.H.E. van Doorn and A.J. Burggraaf, Solid State Ionics 128 (2000) 65

[3] I. Rom et al., Chem. Mater., 14(1) (2002) 135

[4] We gratefully acknowledge financial support by the Fonds zur Förderung der wissenschaftlichen Forschung, Vienna, Austria within the Special Research Program Electroactive Materials. 

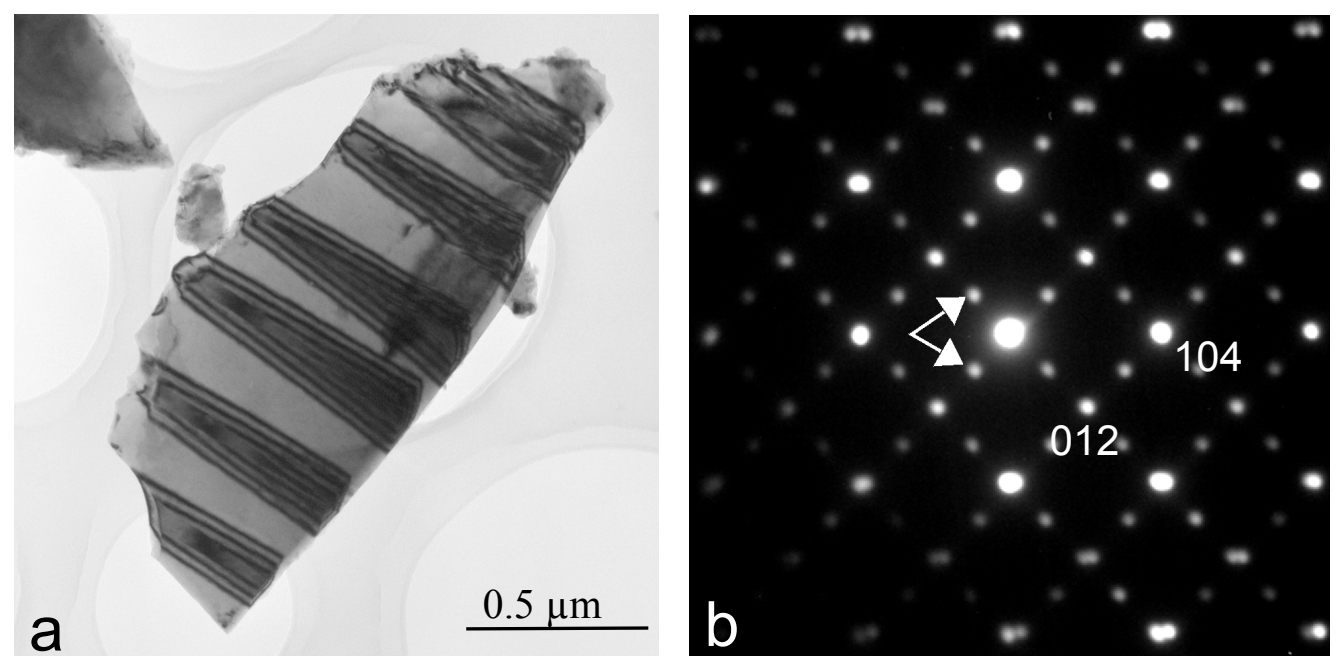

Fig. 1a. TEM bright field image of LSC + , b. SAED pattern with superstructure spots (see arrows) in [4 2 -1] orientation (hexagonal indexing).

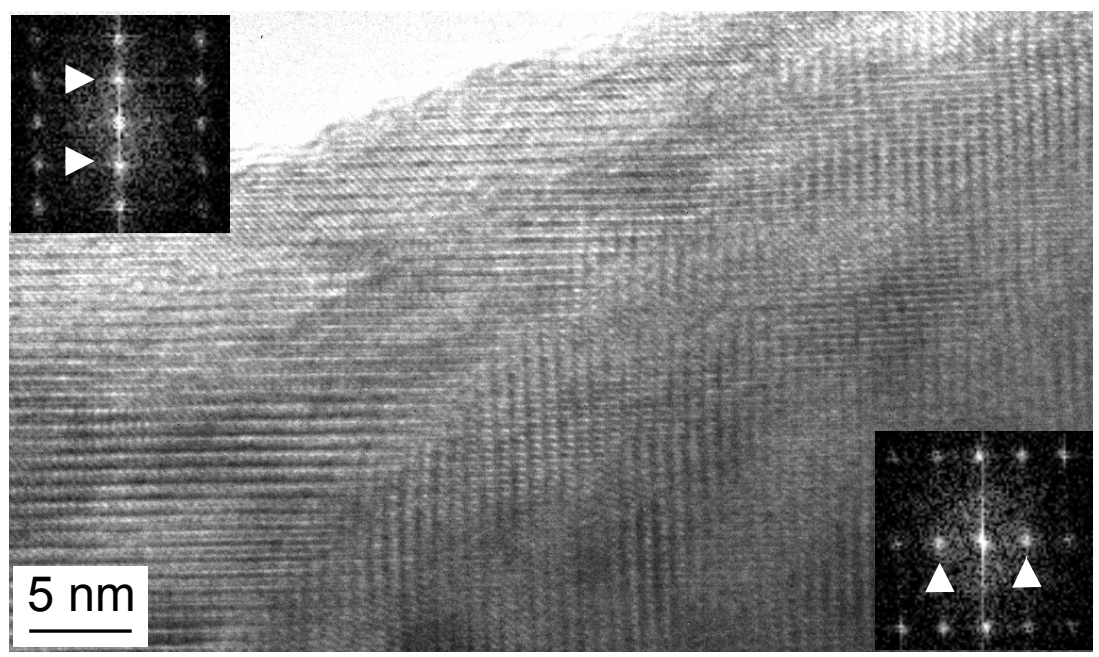

Fig. 2. HRTEM image of an LSC + particle, showing two domains rotated about $90^{\circ}$ with respect to each other. The insets show the corresponding FFTs; spots due to superstructure are marked.
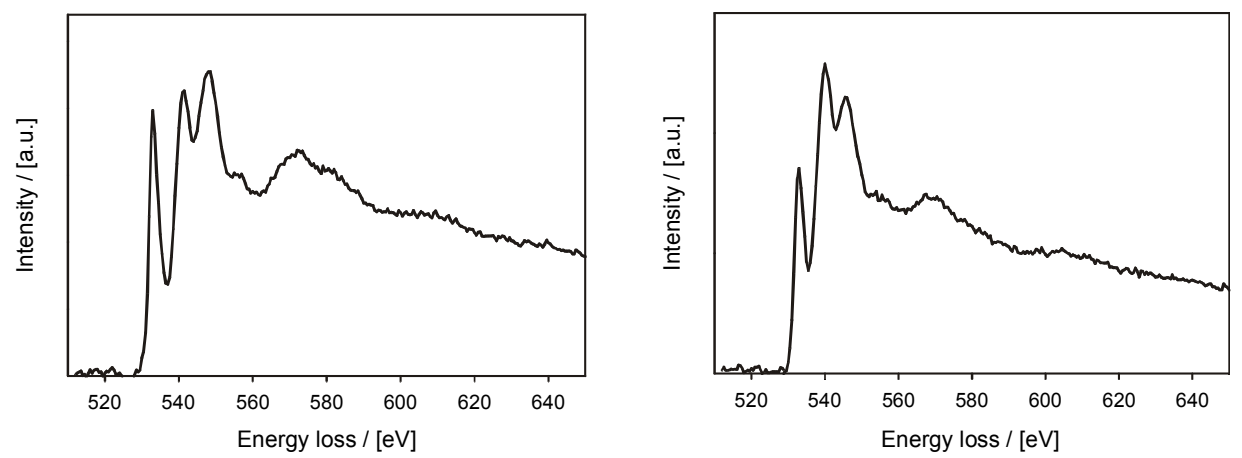

Fig. 3. Near-edge fine structure of the O K ionization edge of LSC (left) and LSC+ (right). 